\title{
Ras-Related Nuclear Protein Ran3B Gene Is Involved in Hormone Responses in the Embryogenic Callus of Dimocarpus longan Lour.
}

\author{
Qilin Tian, Yuling Lin, Dongmin Zhang, Ruilian Lai and Zhongxiong Lai * \\ Institute of Horticultural Biotechnology, Fujian Agriculture and Forestry University, Fuzhou 350002, China; \\ tianqilin2009@163.com (Q.T.); buliang84@163.com (Y.L.); qiandanqianlan2011@163.com (D.Z.); \\ lair10618@163.com (R.L.) \\ * Correspondence: laizx01@163.com; Tel.: +86-591-8378-9484
}

Academic Editor: Jianhua Zhu

Received: 19 March 2016; Accepted: 23 May 2016; Published: 3 June 2016

\begin{abstract}
Ras-related guanosine triphosphate (GTP)-binding nuclear protein (Ran) GTPases function as molecular switches and regulate diverse cellular events in eukaryotes. Our previous work suggested that DlRan3B is active during longan (Dimocarpus longan Lour.) somatic embryogenesis (SE) processes. Herein, subcellular localization of DIRan3B was found to be localized in the nucleus and expression profiling of $D l R a n 3 B$ was performed during longan SE and after exposure to plant hormones (indoleacetic acid (IAA), gibberellin A3 (GA3), salicylic acid (SA), methyl jasmonte (MeJA), and abscisic acid (ABA)). We cloned and sequenced $1569 \mathrm{bp}$ of $5^{\prime}$-flanking sequence of DlRan3B (GenBank: JQ279697). Bioinformatic analysis indicated that the promoter contained plant hormone-related regulatory elements. Deletion analysis and responses to hormones identified stimulative and repressive regulatory elements in the $D l R a n 3 B$ promoter. The key elements included those responding to auxin, gibberellin, SA, MeJA, and ABA. DIRan3B was located in the nucleus and accumulated in the late stage of longan SE. The expression of $D l R a n 3 B$ was significantly induced by IAA, $\mathrm{GA}_{3}$, and ABA, but suppressed by SA and MeJA. Promoter transcription was induced by IAA and $\mathrm{GA}_{3}$, but suppressed by SA. Thus, DlRan3B might participate in auxin, gibberellin, and ABA responses during longan late $\mathrm{SE}$, and $\mathrm{DlRan} 3 B$ is involved in phytohormone responsiveness.
\end{abstract}

Keywords: Ran GTPases; DlRan3B; SE; gene expression; promoter deletion analysis

\section{Introduction}

Ran GTPases regulate a multiple series of cell activities by functioning as molecular switches in animals [1-3]. In plants, Ran GTPases are involved in various responses, including mediating hormone sensitivities [4-6]. Furthermore, overexpression of a Ran GTPase homolog in different plants has caused a variety of developmental abnormalities, such as increases in primordial tissue, mitotic index, sensitivity to exogenous auxin and ABA treatments, and decreases in lateral root number [7-10]. Meanwhile, the mutations in some Ran GTPase homologs can lead to male sterility [9]. These results have led to a conclusion that Ran is essential for plant development and also serve as clues to the potential function of Ran in hormone signaling transduction in plants.

Longan tree (Dimocarpus longan Lour.) is one of the evergreen fruit trees grown in southern China and its fruit have important applications in food industry and health care [11-13]. The development of longan seeds is crucial for longan fruit development, including fruit appearance and processes of fruit setting and ripening. Plant Ran might be involved in the cell activities during plant embryos development, because of its animal its counterparts' tissue-specific expression during embryogenesis and its role in cell division in animal embryos [14-16]. Nevertheless, characterization of plant Ran, especially its involvement in plant embryogenesis and hormone transduction, remains poorly reported. 
In previous studies, full-length cDNAs and DNAs of DlRan3 were cloned from longan somatic embryos and thereafter two $5^{\prime}$ flanking sequences (1256 and 714 bp) of DlRan3A (GenBank: JQ775539) and DlRan3B (GenBank: JQ279697), respectively, were isolated. It was analyzed by bioinformatics that major elements in the promoters were closely related to phytohormones [17]. A previous study also showed DlRan3A's possible participation in auxin signaling transduction in early stages of somatic embryogenesis (SE) in longan and its possible roles in plant hormone, light, and abiotic stress responsiveness. However, to date, little is known about how DlRan3B functions in plant hormone responsiveness in plant embryos.

To further reveal the biological role of DlRan3B, its protein subcellular location was observed and its expression profile was analyzed during longan SE processes and under treatments of exogenous plant hormone, including indoleacetic acid (IAA), gibberellin A3 $\left(\mathrm{GA}_{3}\right)$, salicylic acid (SA), methyl jasmonte (MeJA), and abscisic acid (ABA). Furthermore, to understand the regulatory role of DlRan3B, its $5^{\prime}$ flanking sequence was isolated and characterized to identify an efficient promoter that could initiate the constitutive expression of a foreign gene in transgenic plants. Furthermore, deletion analysis and different transcriptional activities in response to phytohormones showed that the DlRan3B promoter has positive and negative regulatory elements. This study provides a multifaceted view of the potential roles of DlRan3B during longan somatic embryo formation and in phytohormone signaling pathways.

\section{Results}

\subsection{Subcellular Localization of DlRan3B}

A fusion protein of DlRan3B-mGFP (green fluorescent protein) was transiently expressed in the epidermal cells of tobacco leaves to detect subcellular localization of DIRan3B. As a result, DIRan3B-mGFP was dominantly located in the nucleus (Figure 1 and Figure S1).

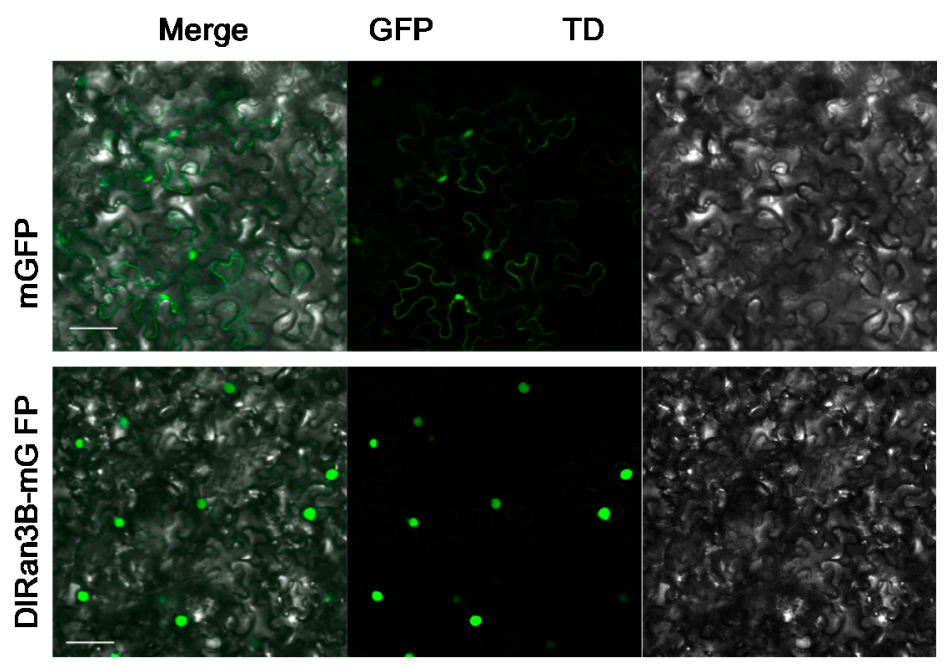

Figure 1. Subcellular localization of DIRan3B. Transient expression of a fluorescent fusion protein (DlRan3B-mGFP (green fluorescent protein)) by agro-infiltration in epidermal cells of tobacco (Nicotiana benthamiana) leaves; the upper image (mGFP) is green fluorescence of pCAMBIA1302-GFP and the lower one (DIRan3B-mGFP) is green fluorescence of DIRan3B-mGFP; TD refers to the transmitted light channel. Bars $=50 \mu \mathrm{m}$.

\subsection{The Expression Profiling of DIRan3B During Longan Somatic Embryogenesis (SE)}

To discover the transcriptional control of DlRan3B during longan SE, we measured the transcript levels of DlRan3B during longan SE. DlRan3B showed decreasing expression during primary developmental stages, with the least DlRan3B accumulation observed in the globular embryo (GE) 
stage, while increasing expression was observed during the middle and late stages of SE, with the highest $D I R a n 3 B$ accumulation in the cotyledon embryo (CE) stage. The distinctive pattern suggested an important role of $D l R a n 3 B$ accumulation during longan late SE, rather than early stages (Figure 2).

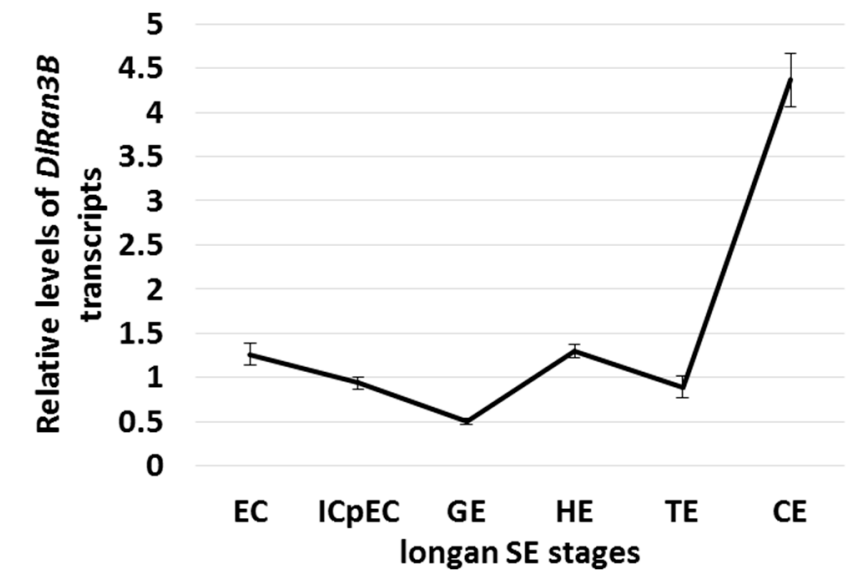

Figure 2. DlRan3B expression pattern in longan somatic embryogenesis (SE). Longan SE stages include embryogenic callus (EC), incomplete compact pro-embryogenic cultures (ICpEC), globular embryo (GE), heart-shaped embryo (HE), torpedo-shaped embryo (TE), and cotyledon embryo (CE). The DIRan3B expression level was normalized to those of EF-1a, eIF-4a, and DLFSD1a. Data are shown as means $\pm \mathrm{SD}(n=3)$.

\subsection{The Effect of Exogenous Plant Hormones on DlRan3B Expression}

To understand how $D l R a n 3 B$ responds to plant hormones, we analyzed the relative levels of DlRan3B mRNA in longan embryogenic calluses (ECs) treated with different concentrations of IAA, $\mathrm{GA}_{3}, \mathrm{SA}, \mathrm{MeJA}$, and ABA (Figure 3A-E). Among the treatments, $26.0 \mu \mathrm{M} \mathrm{GA}$ enhanced the DlRan3B transcript level to approximately 1.6-fold in contrast to the control (Figure 3B); ABA induced a slight, positive transcriptional control on $D l R a n 3 B$, with no dose-dependent effects (Figure 3E). IAA over the range of 2.9-8.6 $\mu \mathrm{M}$ increased $D l \operatorname{Ran} 3 B$ expression, with $8.6 \mu \mathrm{M}$ IAA inducing a 1.3-fold level to the control; by contrast, high concentrations of IAA $(11.4 \mu \mathrm{M})$ inhibited expression (Figure 3A). Notably, DlRan3B gene expression showed a sustained decrease as the SA concentration increased (Figure 3C). In addition, a fluctuating expression pattern was found under MeJA treatment (Figure 3D). These results indicated that the transcription of $D l R a n 3 B$ responded to plant hormones like auxin, gibberellin, SA, MeJA, and ABA.

\subsection{The Isolation and Bioinformatic Analysis of the Putative Promoter Region of the DlRan3B Gene}

To figure out the regulatory roles of $D \operatorname{lRan} 3 B$, we further cloned a 1569-bp 5 -flanking sequence upstream of the $D l R a n 3 B$ translation initiation site (ATG), covering a previously cloned $5^{\prime}$-flanking sequence of DlRan3B (714 bp, GenBank: JQ279697). Thereafter, the Berkeley Drosophila Genome Project and PlantCARE databases were used to predict and analyze potential core sequences and regulatory elements of $D l R a n 3 B$ promoter region. Two core sequences in the promoter region were predicted at -952 to -903 (score 0.87 ) and at -79 to -30 (score 0.97 ) upstream of the ATG. The potential transcription start sites (TSSs) were T and C, respectively. In the previous study [18], some of the TSSs were proved to be in the region between -79 and -30 , but not in the sequence between -952 and -903 , thus we proposed that the actual core sequence within the 1569-bp promoter region of DlRan3B was located in the region from -79 to -30 and the actual TSS was C (Figure 4). Further analysis identified conserved TATA and CAAT boxes dispersed over the entire promoter sequence. The most frequent motifs observed in the $D l R a n 3 B$ promoter were elements involved in light and hormone responses (auxin, gibberellin, SA, MeJA, and ABA). Stress-responsive motifs were also found 
throughout the promoter region, for example, those involved in low-temperature responsiveness and drought-inducibility (Figure 4, Table 1).

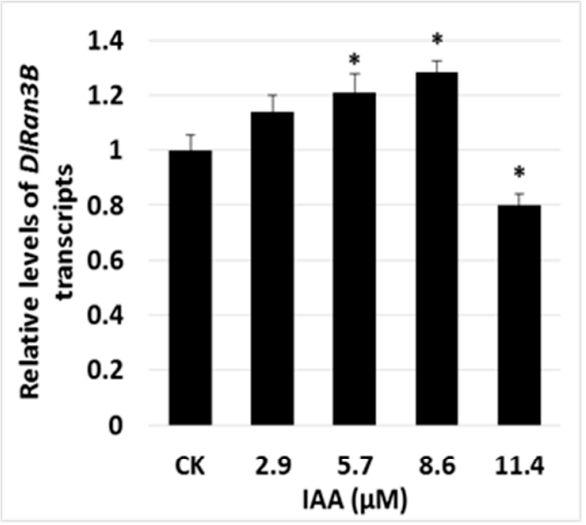

(A)

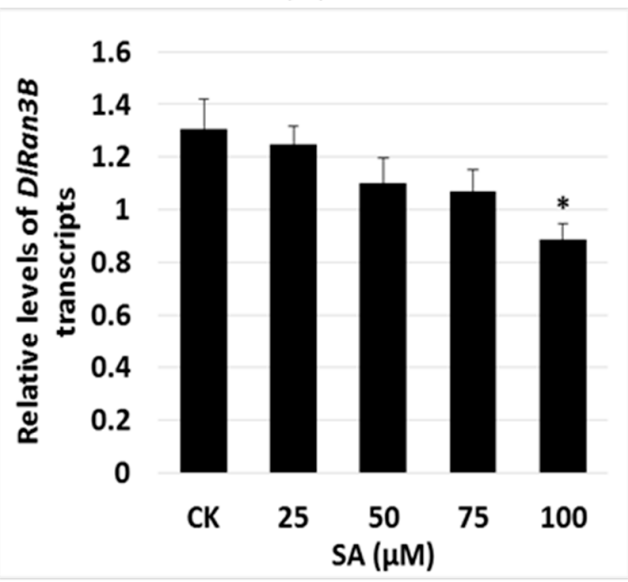

(C)

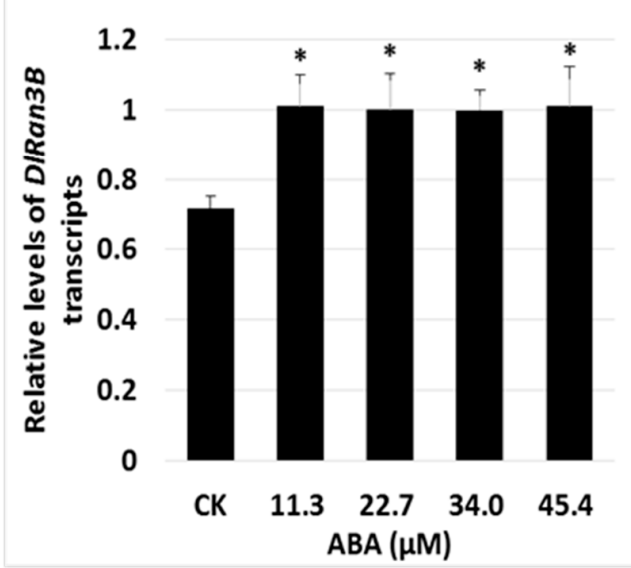

(E)

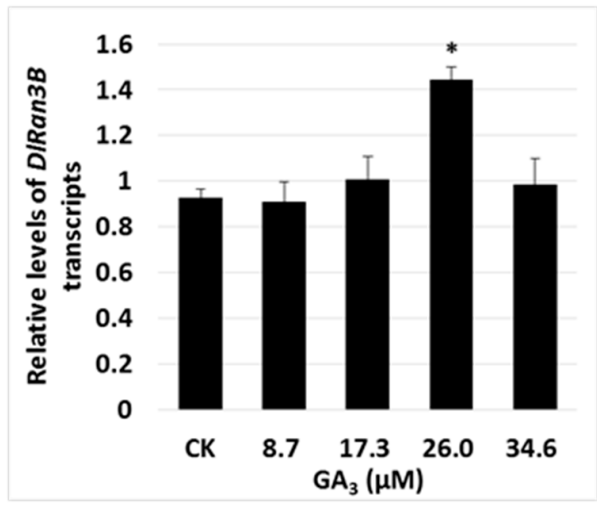

(B)

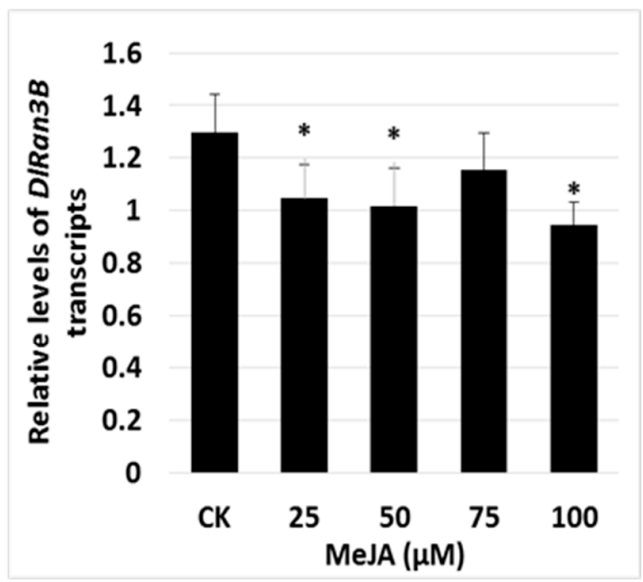

(D)

Figure 3. DlRan3B expression in response to exogenous plant hormones. (A-E) ECs were treated with listed hormones at indicated concentrations as follows, (A) indoleacetic acid (IAA) $(2.9,5.7,8.6$, and $11.4 \mu \mathrm{M})$; (B) gibberellin A3 (GA3) (8.7, 17.3, 26.0, and $34.6 \mu \mathrm{M})$; (C) salicylic acid (SA) (25, 50, 75, and $100 \mu \mathrm{M})$; (D) methyl jasmonte (MeJA) (25, 50, 75, and $100 \mu \mathrm{M})$; or (E) abscisic acid (ABA) (11.3, 22.7, 34.0, and $45.4 \mu \mathrm{M})$. The DIRan3B expression level was normalized to those of $E F-1 a$, eIF-4a, and DLFSD1a. Data are shown as means $\pm \mathrm{SD}(n=3)$; the level of significant differences in contrast to the control (CK) are indicated with an asterisk $\left(^{*}\right)$ and were assessed by a one-way ANOVA test $\left({ }^{*} p<0.05\right)$. 


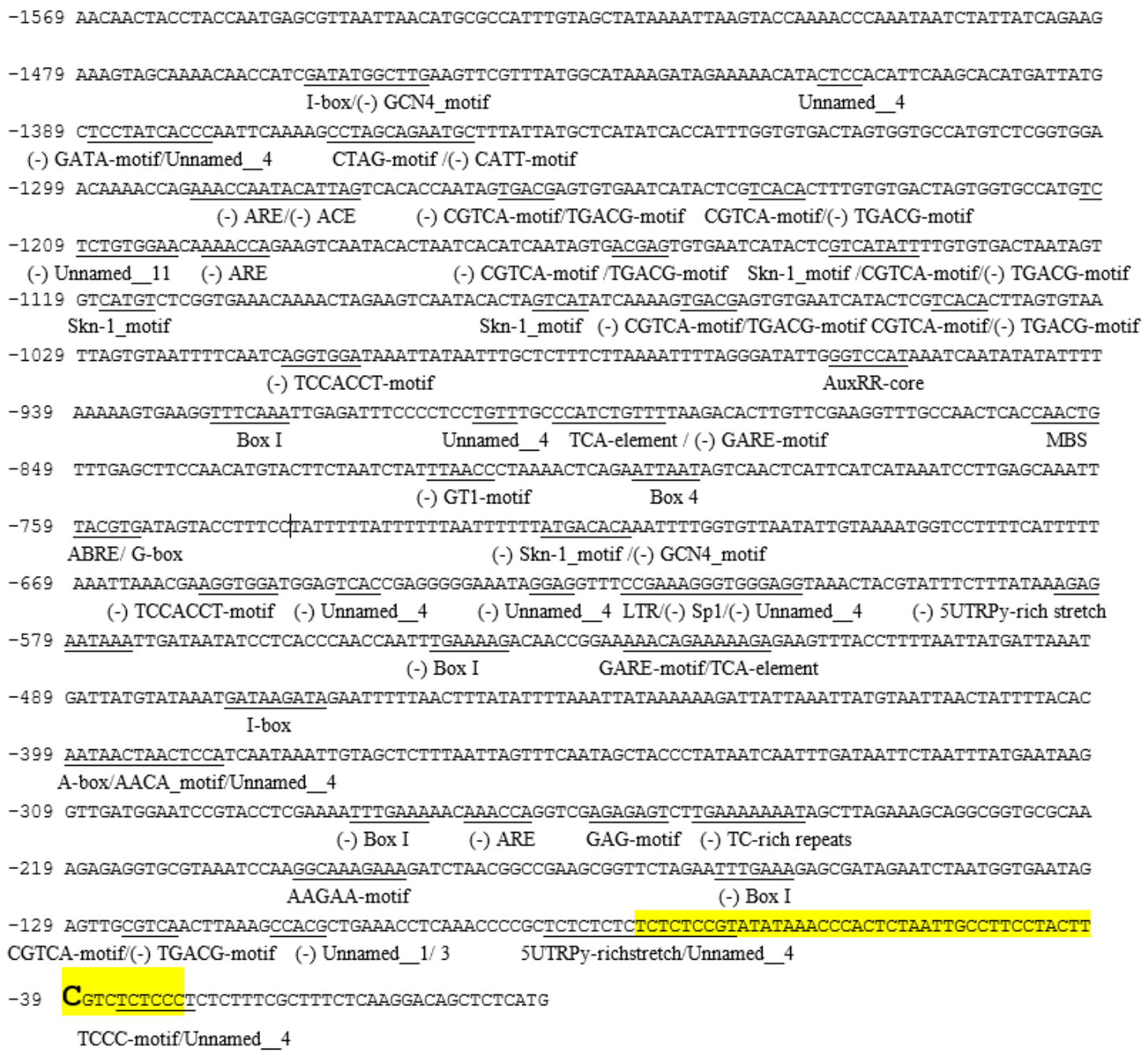

Figure 4. The sequence and structure analysis of the promoter of DlRan3B. The translation initiation site is ATG, the core promoter region is highlighted in yellow, and the transcription start sites (TSS) is highlighted in large bold letters.

\subsection{Deletion Analysis of the DIRan3B Promoter}

To detect the transcription regulation of the $D l R a n 3 B$ promoter and figure out key regulatory regions, the constructs of the 1569-bp flanking fragment and a variety of $5^{\prime}$ and $3^{\prime}$ deletions, all fused to GUS, a promoterless reporter gene, were prepared (Figure 5). Quantitative real-time PCR (qPCR) assays showed that all of the constructs expressed in tobacco leaves produced relatively lower levels of GUS transcripts compared with the control (CaMV35S, 35S) (Figure 5). The difference of GUS transcripts between each of the two $5^{\prime}$ deletions showed that activation functional elements were distributed in the positions from -1269 to -924 and -616 to -558 , while repression functional elements existed at positions from -1569 to -1269 and -858 to -616 . Among the $3^{\prime}$ deletions, positive elements were distributed in the regions from -1211 to -863 and -245 to -1 , while inhibitory ones were at -863 to -245 . It indicated that both positive and negative functional elements exist in the DlRan3B promoter region. 
Table 1. Prediction of regulatory elements in the $5^{\prime}$-flanking sequence of DlRan3B.

\begin{tabular}{|c|c|c|}
\hline Regulatory Element & Function & Sequence \\
\hline 5UTR Py-rich stretch & cis-acting element conferring high transcription levels & ТTТСТТСТСТ/ТТТСТСТСТСТСТС \\
\hline A-box & sequence conserved in alpha-amylase promoters & AATAACAAACTCC \\
\hline AACA-motif & involved in endosperm-specific negative expression & TAACAAACTCCA \\
\hline ABRE & cis-acting element involved in the abscisic acid responsiveness & TACGTG \\
\hline ARE & cis-acting regulatory element essential for the anaerobic induction & TGGTTT \\
\hline $\mathrm{ACE}$ & cis-acting regulatory element involved in light responsiveness & CTAACGTATT \\
\hline AuxRR-core & cis-acting regulatory element involved in auxin responsiveness & GGTCCAT \\
\hline Box 4 & part of a conserved DNA module involved in light responsiveness & ATTAAT \\
\hline Box I & light responsive element & TTTCAAA \\
\hline CAAT-box & common cis-acting element in promoter and enhancer regions & CAAT/CAAAT \\
\hline CGTCA-motif & cis-acting regulatory element involved in the MeJA-responsiveness & CGTCA \\
\hline G-box & cis-acting regulatory element involved in light responsiveness & CACGTA \\
\hline GAG-motif & part of a light responsive element & AGAGAGT \\
\hline GARE-motif & gibberellin-responsive element & AAACAGA \\
\hline GATA-motif & part of a light responsive element & GATAGGA \\
\hline GCN4-motif & cis-acting element involved in endosperm expression & CAAGCCA/TGTGTCA \\
\hline GT1-motif & light responsive element & GGTTAA \\
\hline I-box & part of a light responsive element & GATATGG/GATAAGATA \\
\hline LTR & cis-acting element involved in low-temperature responsiveness & CCGAAA \\
\hline MBS & v-myb avian myeloblastosis viral oncogene homolog (MYB) binding site involved in drought-inducibility & CAACTG \\
\hline Skn-1-motif & cis-acting regulatory element required for endosperm expression & GTCAT \\
\hline Sp1 & light responsive element & $\mathrm{CC}(\mathrm{G} / \mathrm{A}) \mathrm{CCC}$ \\
\hline TATA-box & core promoter element around -30 of transcription start & TATA/TAATA/TTTTA \\
\hline TC-rich repeats & cis-acting element involved in defense and stress responsiveness & ATTTTCTTCA \\
\hline TCA-element & cis-acting element involved in salicylic acid responsiveness & ССАТСТTTTT/CAGAAAAGGA \\
\hline TCCC-motif & part of a light responsive element & TCTCCCT \\
\hline TGACG-motif & cis-acting regulatory element involved in the MeJA-responsiveness & TGACG \\
\hline
\end{tabular}




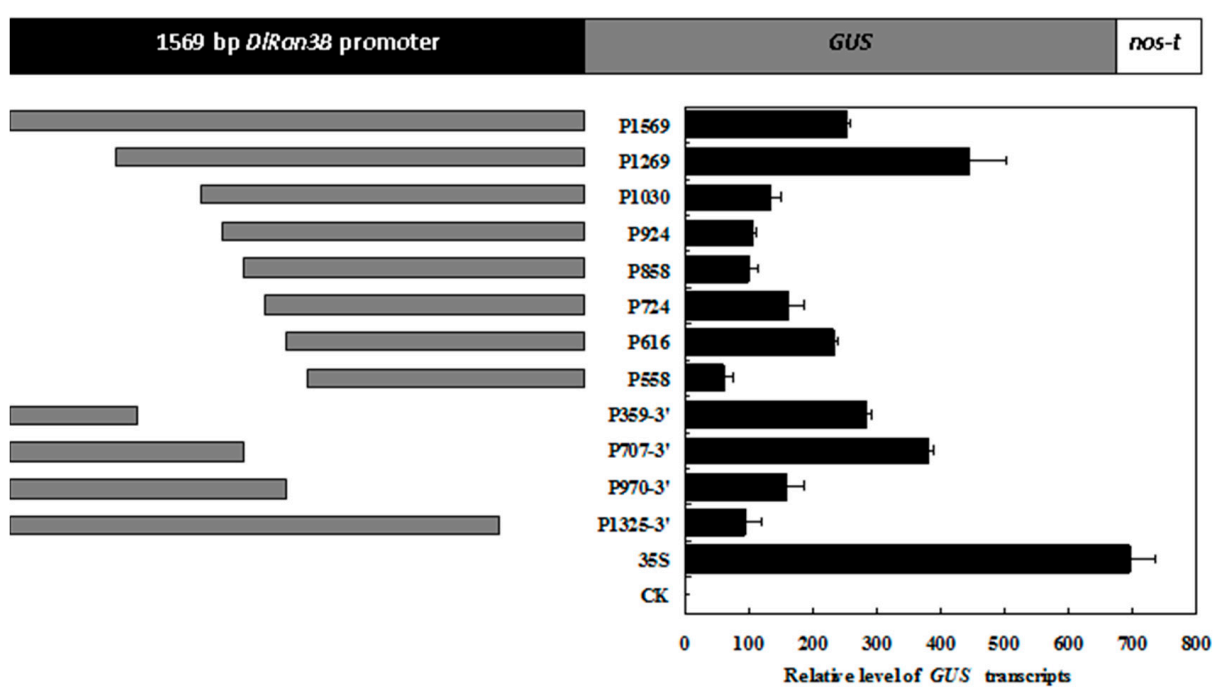

Figure 5. Schematic diagram of the DlRan3B promoter deletion constructs. 35 S represents the samples with pBI121 vector, and CK represents wild-type tobacco. The GUS expression level was normalized to 18 SrRNA. Data are shown as means $\pm \operatorname{SD}(n=3)$.

\subsection{Responsiveness of the DlRan3B Promoter to Hormone Treatments}

The DlRan3B promoter contains certain phytohormone-responsive elements, such as ones involved in auxin (AuxRR-core), gibberellin (GARE-motif), SA (TCA-element), MeJA (TGACG- and CGTCA-motif), and ABA (ABRE) responsiveness. To detect the effects of plant hormones on the transcription regulation of DlRan3B promoter, $8.6 \mu \mathrm{M}$ IAA, $34.6 \mu \mathrm{M} \mathrm{GA}_{3}, 75 \mu \mathrm{M} \mathrm{SA}, 100 \mu \mathrm{M}$ MeJA, and $75.7 \mu \mathrm{M}$ ABA were used in treating tobacco leaves harboring the construct of DlRan3B promoter. The highest GUS expression was found in samples under IAA treatment, which showed more than 3-fold higher GUS expression compared with the control. GA G $_{3}$ also induced GUS expression, by about 1.5-fold higher than the control. By contrast, GUS expression in the SA-treated samples was repressed. These data demonstrated that regulatory elements related to auxin, gibberellin, and SA responsiveness co-exist in the 1569-bp region of the DlRan3B promoter and they are involved in regulating the transcription of a target gene; meanwhile, MeJA and ABA had no significant effect on the transcription regulation of the DlRan3B promoter (Figure 6).

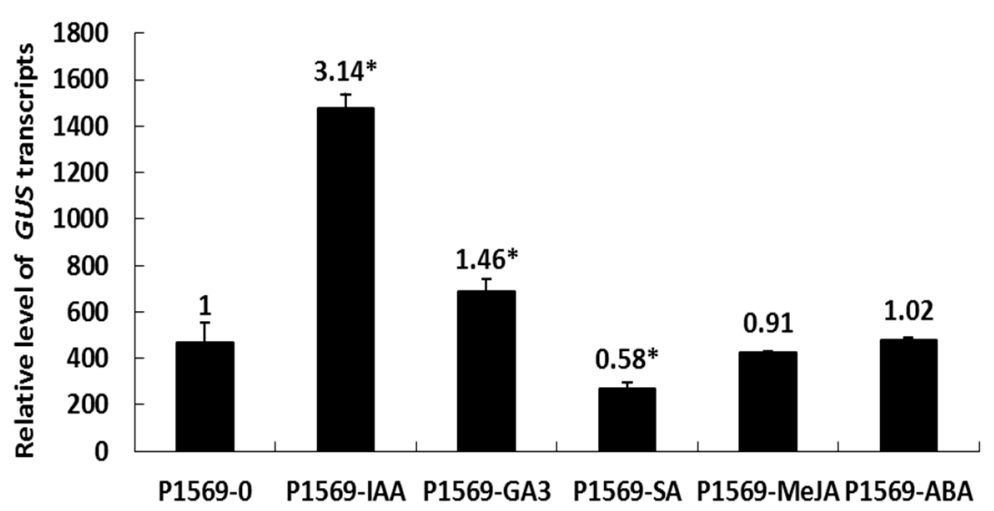

Figure 6. Response of the DlRan3B promoter to plant hormones. Tobacco leaves infiltrated with the construct P1569 were under the treatments of $8.6 \mu \mathrm{M}$ IAA, $34.6 \mu \mathrm{M} \mathrm{GA}_{3}, 75 \mu \mathrm{M} \mathrm{SA}, 100 \mu \mathrm{M}$ MeJA, $75.7 \mu \mathrm{M}$ ABA, and water, as a control. Data are shown as means $\pm \mathrm{SD}(n=3)$. Numbers over the bars represent the fold induction of the five plant hormone treatments over the control; the level of significant differences in contrast to the control (P1569-0) are indicated with an asterisk $\left({ }^{*}\right)$ and were assessed by a one-way ANOVA test $(* p<0.05)$. 


\section{Discussion}

\subsection{DlRan3B Shares Similar Subcellular Localization with Its Longan Homolog and Animal Counterpart}

The result of the subcellular localization assay revealed that DIRan3B was exclusively located in the nucleus (Figure 1 and Figure S1), slightly different from DIRan3A, another member in the longan Ran family, which was primarily located in the nucleus, with some in the cytoplasm [6]. The nuclear location of DlRan3B is similar with its longan homolog DlRan3A and animal counterpart. Ran is essential for transporting proteins through the nuclear pore in yeast and mammalian tissue [19,20]. DIRan3B might have a similar nucleocytoplasmic trafficking function to its animal counterparts. However, it remains to be verified what is the relationship between DlRan3Bs accumulation and longan late SE, and how DIRan3B functions in embryogenic cells.

\subsection{DlRan3B Participates in the Auxin, Gibberellin, and ABA Signaling Pathways During Longan Late SE}

In the early courses of longan $\mathrm{SE}$, there are high levels of total auxin and gibberellin (IAA + GA 3 ), while longan late SE is associated with lower levels of IAA $+\mathrm{GA}_{3}$. There was a slight increase in endogenous $\mathrm{ABA}$ as SE progressed in longan; during late SE there was a sharp rise in the ratio of $\mathrm{ABA} /\left(\mathrm{IAA}+\mathrm{GA}_{3}\right)$ to a maximum, rather than a distinct increase in the absolute level of $\mathrm{ABA}$, which suggested the critical role of ABA in the late development of longan somatic embryos [21]. Here, we found that lower doses of IAA and $\mathrm{GA}_{3}$ increased the expression of $D l R a n 3 B$, while higher doses did not. Therefore, the low levels of DlRan $3 B$ transcripts in early longan SE could be attributed to the high levels of auxin and gibberellin. By contrast, DlRan $3 B$ expression increased to its maximum at the CE stage, probably because of the complex effects of IAA $+\mathrm{GA}_{3}$ and ABA. This was different to the expression pattern observed for $D l \operatorname{Ran} 3 A$ [6], and suggested that $D l R a n 3 B$ plays a more important role in longan late $\mathrm{SE}$, involving the hormone signaling pathways of auxin, gibberellin, and ABA. However, during longan SE, the exact nature of this complex relationship among auxin, gibberellin, and ABA has not yet been determined.

\subsection{The Potential Role of DlRan3B}

Ran and Ran-binding proteins have important roles in actively transporting various light and phytohormone receptors, transcription factors, and signaling regulatory proteins into the nucleus when plant cells are exposed to light or phytohormones [4]. However, little is uncovered about the roles of phytohormone in the regulatory network of the Ran gene. Similar to DlRan3A [6], in the DlRan3B's 5' flanking sequence, there are many elements involved in light responsiveness (ACE, Box 4, Box I, G-box, GAG-motif, GATA-motif, GT1-motif, I-box, Sp1, and TCCC-motif) and elements involved in hormone responsiveness (ABRE, AuxRR-core, CGTCA-motif, GARE-motif, TCA-element, and TGACG-motif). Here, we found that certain concentrations of IAA and $\mathrm{GA}_{3}$ increased DlRan3B expression and its promoter transcription activity, which suggested that $D l R a n 3 B$ might participate in auxin and gibberellin responsiveness. The auxin-responsive element is located at -1211 to -924 of the DlRan3B promoter region and might be a critical regulatory element for stimulating DlRan $3 B$ expression. A novel role of Ran in meristem initiation regulated by auxin signal was observed by overexpressing TaRAN1 in transgenic Arabidopsis and rice, and a close association between Ran and auxin was also discovered in Ran-related proteins $[7,22]$. Some reports have shown that exogenous $\mathrm{GA}_{3}$ upregulates the expression of transcription factors for embryogenesis, and induces the regeneration of somatic embryos, with the acceleration of starch hydrolysis by enhancing $\alpha$-amylase activity [23,24]. Therefore, DlRan3B's response to $\mathrm{GA}_{3}$ and the existence of an A-box (a sequence conserved in alpha-amylase promoters) in the $D l R a n 3 B$ promoter suggest the probable involvement of gibberellin in activating nucleocytoplasmic transport of signal elements associated with energy metabolism during longan SE. Nevertheless, little direct evidence is available about the relationship between Ran and hormone signaling pathways and about how Ran and its promoter function in plant somatic embryos. 
The crucial functions of the nuclear trafficking machinery in plant immune and stress signals were reviewed by García and Parker [5]. SA plays a key role in abiotic stress responses, including drought, low temperature, and salinity responsiveness. Some reports have demonstrated that the Ran protein is involved in abiotic stress responses, such as mechanical wounding [25], osmotic stress [9], salinity [26], and cold stress $[10,27]$. The observation that SA repressed DlRan3B expression and its promoter transcription activity correlates with the coexistence of elements involved in drought-inducibility and low-temperature responsiveness in the DlRan $3 B$ promoter region -858 to -616 , which might be negative regulatory elements. Therefore, we hypothesized that SA might participate in the complex interaction of defense-related cis-acting elements and trans-acting factors, and the transport of certain transcription factors or mRNA through the nuclear pore via its effect on the DlRan3B promoter. In addition, the fact that DIRan3B shares ARE (elements involved in anaerobic induction) and TC-rich repeats (elements involved in defense and stress responses) with DlRan $3 A$ in its promoter region suggested that the longan Ran gene family might participate in the response to environmental stress by the mutual effects of different family members. Thus, the ARE, TC-rich repeats, MBS (drought-inducibility), LTR (low-temperature responsiveness), TCA-element (SA responsiveness), and some light-responsive elements, which were located in -1569 to $-1269,-858$ to -616 , and -558 to -245 , might be negative regulatory elements. The AuxRR-core (auxin responsiveness), $5^{\prime} U T R$ Py-rich stretch (high transcription levels conferring), and some other light-responsive elements, which were located at -1211 to $-924,-616$ to -558 , and -245 to -1 , might be positive regulatory elements. The MeJA-responsiveness elements might be involved in complex activation and repression regulation because of their scattered pattern in the promoter region. With environmental stimuli, Ran's roles in cell activities and nucleocytoplasmic transport of signal elements in plant embryos require further research.

\section{Materials and Methods}

\subsection{Plant Materials and Nucleic Acid Extraction}

Synchronized longan embryogenic cultures from six longan SE stages (i.e., EC, incomplete compact pro-embryogenic cultures (ICpEC), GE, heart-shaped embryo (HE), torpedo-shaped embryo (TE) and CE) were obtained using previously published methods [28,29]. EC were kept on Murashige and Skoog (MS, Phytotechnology M519) medium (2\% sucrose, $6 \mathrm{~g} / \mathrm{L}$ agar, $\mathrm{pH} 5.8$ ) supplemented with $4.5 \mu \mathrm{M}$ 2,4-Dichlorophenoxyacetic acid (2,4-D), and MS medium supplemented with $4.5 \mu \mathrm{M} 2,4-\mathrm{D}$, $2.3 \mu \mathrm{M}$ kinetin, and $5 \mathrm{mg} / \mathrm{L} \mathrm{AgNO}_{3}$, subcultured every $20 \mathrm{ds}$, alternatively. Synchronized GE and the other four embryogenic cultures were obtained by transferring EC to MS medium supplemented with $0.45 \mu \mathrm{M} 2,4-\mathrm{D}$ and MS medium, respectively. All cultures were kept in the dark and at the temperature of $25^{\circ} \mathrm{C}$, with more than three replicates. Tobacco (K326 and Nicotiana benthamiana) plants were grown in a growth chamber at $25^{\circ} \mathrm{C}$ under a $16 / 8$-h photoperiod $\left(100 \mu \mathrm{mol} / \mathrm{m}^{2} / \mathrm{s}\right)$. Genomic DNA and total RNA were extracted from the above-mentioned samples as previously described [6,17].

\subsection{Subcellular Localization}

The subcellular localization assay was conducted as previously described [6]. DlRan3B cDNA was fused to the N-terminus of the GFP in the plasmid of pCAMBIA1302. Transient transformation of fluorescent fusion protein by agro-infiltration in epidermal cells of tobacco (Nicotiana benthamiana) leaves was performed as previously executed and the subcellular localization of DIRan3B-mGFP was analyzed by laser scanning confocal microscopy (Olympus (Tokyo, Japan); FV1200) [6] .

\subsection{Phytohormone Treatments on Longan EC Samples}

After subculturing for 18 days, longan EC $(0.2 \mathrm{~g})$ was transferred to 40-mL MS liquid medium ( $2 \%$ sucrose) supplemented with IAA $(2.9,5.7,8.6$, and $11.4 \mu \mathrm{M}), \mathrm{GA}_{3}(8.7,17.3,26.0$, and $34.6 \mu \mathrm{M})$, SA $(25,50,75$, and $100 \mu \mathrm{M})$, MeJA $(25,50,75$, and $100 \mu \mathrm{M})$, and $\mathrm{ABA}(11.3,22.7,34.0$, and $45.4 \mu \mathrm{M})$, under agitation at $120 \mathrm{rpm}$ at $25^{\circ} \mathrm{C}$ under dark for $24 \mathrm{~h}$, with three replicates. Controls were EC transferred 
to MS liquid medium. All samples of the above-mentioned treatments were frozen in liquid nitrogen immediately and stored at $-80^{\circ} \mathrm{C}$ for RNA extraction.

\subsection{Isolation of DlRan3B Promoter and Bioinformatic Analysis}

Genome walking technique was conducted by thermal asymmetric interlaced PCR (Tail-PCR, Takara, Otsu, Japan) to acquire a longer DlRan3B 5'-flanking sequence. Each of the three nested PCR amplifications used forward primer AP (AP1, AP2, AP3, or AP4) and specific reverse primers (DIRan3B-pro2-SP1: CGGATTCCATCAACCTTATTCATAAATTAG, DIRan3B-pro2-SP2: GTTGTCT TTTCAAATTGGTTGGGTGAGG, and DIRan3B-pro2-SP3: CGTAGTTTACCTCCCACCCTTTCGG) to obtain the flanking sequence of DlRan3B. The cycling conditions of all PCR amplifications were similar to those of Lin's protocol [30], with some modifications. The potential core promoter sequences and functional elements of the DlRan3B promoter were predicted by the Berkeley Drosophila Genome Project and PlantCARE databases [31].

\subsection{Construction of the PdlRan3B::GUS Fusion Vector and Agrobacterium-Mediated Transient Assay}

Agrobacterium-mediated transient transformation of epidermal cells in tobacco leaves is a relatively fast technique to determine expression of genes of interest [32]. The $1569 \mathrm{bp}$ of the flanking region, a series of nested $5^{\prime}$ deletions of the PdlRan3B fragments (1269, 1030, 924, 858, 724, 616, and $558 \mathrm{bp}$ ), and nested $3^{\prime}$ deletions of the PdlRan3B fragments (1325, 970, 707, and $359 \mathrm{bp}$ ) were amplified from the DlRan3B promoter sequence (GenBank accession No. JQ279697) using longan DNA as the template. Longan DNA was extracted from EC using the modified cetyl trimethylammonium bromide method [33]. Forward primers, Del-F1, Del-F2, Del-F3, Del-F4, Del-F5, Del-F6, Del-F7, and Del-F8, were designed to correspond to the $1569,1269,1030,924,858,724,616$, and 558 bp sequences of the $5^{\prime}$ deletions (P1569, P1269, P1030, P924, P858, P724, P616, and P558) and the reverse primer Del-R1 was located in the $3^{\prime}$ end of the DlRan3B promoter (Table 2). Reverse primers, Del-R2, Del-R3, Del-R4, and Del-R5, plus Del-F1, were designed to correspond to the 1325, 970, 707, and 359-bp sequences of the $3^{\prime}$ deletions (P1325-3', P970-3', P707-3', and P359-3') (Table 2). HindIII and BamHI restriction enzyme sites (underlined in Table 2) were introduced at the $5^{\prime}$ end of each forward and reverse primer, respectively. The ligation, Agrobacterium transformation, and Agrobacterium-mediated transient assay in tobacco were conducted as described previously [6].

Table 2. Sequences of primers used to amplify the DlRan3B promoter deletion constructs.

\begin{tabular}{|c|c|c|c|}
\hline Primer & Sequence $\left(5^{\prime}\right.$ to $\left.3^{\prime}\right)$ & Corresponding Construct & Transgenic Line \\
\hline Del-F1 & TGATTACGCCAAGCTTAACAACTACCTACCAATGAGCG & Full-length (P1569) & P1569 \\
\hline Del-R1 & GACCACCCGGGGATCCGAGAGCTGTCCTTGAGAAAGCG & Full-length (P1569) & P1569 \\
\hline Del-F2 & TGATTACGCCAAGCTTCCAATAGTGACGAGTGTGAATC & $5^{\prime} \Delta(\mathrm{P} 1269)$ & P1269 \\
\hline Del-F4 & TGATTACGCCAAGCTTCAAATTGAGATTTCCCСTCCTG & $5^{\prime} \Delta(\mathrm{P} 924)$ & P924 \\
\hline Del-F5 & TGATTACGCCAAGCTTCACCAACTGTTTGAGCTTCCAAC & $5^{\prime} \Delta(\mathrm{P} 858)$ & P858 \\
\hline Del-F6 & TGATTACGCCAAGCTTTTTTTTATGACACAAATTTTGGTG & $5^{\prime} \Delta(\mathrm{P} 724)$ & P724 \\
\hline Del-F7 & TGATTACGCCAAGCTTAGGGTGGGAGGTAAACTACG & $5^{\prime} \Delta(\mathrm{P} 616)$ & P616 \\
\hline Del-R3 & GACCACCCGGGGATCCAGTTTACCTCCCACCCTTTCG & $3^{\prime} \Delta\left(\mathrm{P} 970-3^{\prime}\right)$ & P970-3' \\
\hline Del-R4 & GACCACCCGGGGATCCGGCAAACCTTCGAACAAGTGTC & $3^{\prime} \Delta\left(\mathrm{P} 707-3^{\prime}\right)$ & P707-3' \\
\hline Del-R5 & GACCACCCGGGGATCCACATGGCACCACTAGTCACAC & $3^{\prime} \Delta\left(\mathrm{P} 359-3^{\prime}\right)$ & P359-3' \\
\hline
\end{tabular}

\subsection{Phytohormone Treatments of Tobacco Leaves}

To examine the effects of phytohormone on the transcription regulation of the DlRan3B promoter, tobacco leaves infiltrated with Agrobacterium harboring DlRan3B promoter were sprayed with $8.6 \mu \mathrm{M}$ IAA, $34.6 \mu \mathrm{M} \mathrm{GA}_{3}, 75 \mu \mathrm{M} \mathrm{SA}, 100 \mu \mathrm{M}$ MeJA, or $75.7 \mu \mathrm{M} \mathrm{ABA}$ for $48 \mathrm{~h}$, with sterile water as a control. All tobacco plants were grown and sampled as above described. 


\section{7. $q P C R$ Analysis}

qPCR was conducted to investigate the transcript levels of the DlRan3B gene during longan SE, under a series of plant hormone treatments, and transient expression of the GUS gene in agro-infiltrated tobacco leaf samples, as described above. Total RNA extraction, determination, and cDNA synthesis were conducted as described previously [6]. qPCR of the DlRan3B gene was performed using gene-specific primers (F: CATCATGAAGCTGAGCTTGC; R: CCAGCCTGCAACTGTTCTC), with $E F-1 \alpha, e l F-4 \alpha$, and DlFSD1 $\alpha$ as the reference genes [30,34]. Relative transcript levels of the GUS gene were detected using primers (F: CCTGCGTCAATGTAATGTTCTG; R: TTCTCTGCCGTTTCCAAATC), with 18SrRNA as the reference gene of 18SrRNA (F: CCTGAGAAACGGCTACCACAT; R: CACCAGACTTGCCCTCCA).

Supplementary Materials: Supplementary materials can be found at http:/ /www.mdpi.com/1422-0067/17/ 6/873/s1.

Acknowledgments: This work was funded by the National Natural Science Foundation of China (31572088, 31272149 and 31201614), the Major Science and Technology Program of Fujian Province (2015NZ0002), and the Fujian Provincial Natural Science Foundation of China (2015J06004).

Author Contributions: Qilin Tian participated in the study design, carried out the experiments, and wrote the manuscript. Yuling Lin participated in the study design, provided experimental guidance, and helped draft the manuscript. Dongmin Zhang and Ruilian Lai helped carry out the experiments. Zhongxiong Lai conceived the study, participated in its design and coordination, and helped draft the manuscript.

Conflicts of Interest: The authors declare no conflict of interest.

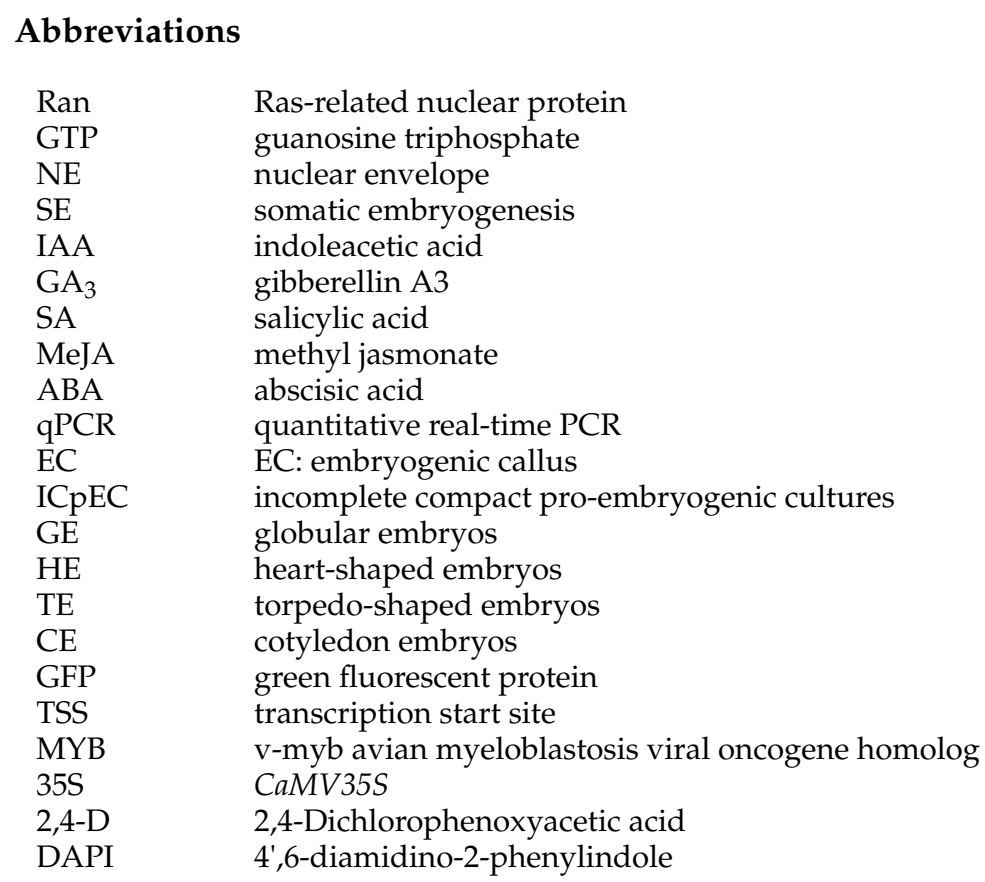

\section{References}

1. Vernoud, V.; Horton, A.C.; Yang, Z.; Nielsen, E. Analysis of the small GTPase gene superfamily of Arabidopsis. Plant Physiol. 2003, 131, 1191-1208. [CrossRef] [PubMed]

2. Saxena, S.; Gandhi, A.; Lim, P.-W.; Relles, D.; Sarosiek, K.; Kang, C.; Chipitsyna, G.; Sendecki, J.; Yeo, C.J.; Arafat, H.A. RAN GTPase and osteopontin in pancreatic cancer. Pancreat. Disord. Ther. 2013, 3, 113. [CrossRef] [PubMed]

3. Hao, W.; Collier, S.M.; Moffett, P.; Chai, J. Structural basis for the interaction between the potato virus X resistance protein (Rx) and its cofactor Ran GTPase-activating protein 2 (RanGAP2). J. Biol. Chem. 2013, 288, 35868-35876. [CrossRef] [PubMed] 
4. $\quad$ Lee, Y.; Lee, H.-S.; Lee, J.-S.; Kim, S.-K.; Kim, S.-H. Hormone-and light-regulated nucleocytoplasmic transport in plants: Current status. J. Exp. Bot. 2008, 59, 3229-3245. [CrossRef] [PubMed]

5. García, A.V.; Parker, J.E. Heaven's gate: Nuclear accessibility and activities of plant immune regulators. Trends Plant Sci. 2009, 14, 479-487. [CrossRef] [PubMed]

6. Tian, Q.; Lin, Y.; Yang, M.; Zhang, D.; Lai, R.; Lai, Z. DlRan3A is involved in hormone, light, and abiotic stress responses in embryogenic callus of Dimocarpus longan Lour. Gene 2015, 569, 267-275. [CrossRef] [PubMed]

7. Wang, X.; Xu, Y.; Han, Y.; Bao, S.; Du, J.; Yuan, M.; Xu, Z.; Chong, K. Overexpression of RAN1 in rice and Arabidopsis alters primordial meristem, mitotic progress, and sensitivity to auxin. Plant Physiol. 2006, 140, 91-101. [CrossRef] [PubMed]

8. Lü, S.; Fan, Y.; Jin, C. Overexpression of a Ran GTPase homologous gene, FaRan from tall fescue, in transgenic Arabidopsis. Biol. Plant. 2011, 55, 331-334. [CrossRef]

9. Zang, A.; Xu, X.; Neill, S.; Cai, W. Overexpression of OsRAN2 in rice and Arabidopsis renders transgenic plants hypersensitive to salinity and osmotic stress. J. Exp. Bot. 2010, 61, 777-789. [CrossRef] [PubMed]

10. Chen, N.; Xu, Y.; Wang, X.; Du, C.; Du, J.; Yuan, M.; Xu, Z.; Chong, K. OsRAN2, essential for mitosis, enhances cold tolerance in rice by promoting export of intranuclear tubulin and maintaining cell division under cold stress. Plant Cell Environ. 2011, 34, 52-64. [CrossRef] [PubMed]

11. Prasad, K.N.; Hao, J.; Shi, J.; Liu, T.; Li, J.; Wei, X.; Qiu, S.; Xue, S.; Jiang, Y. Antioxidant and anticancer activities of high pressure-assisted extract of longan (Dimocarpus longan Lour.) fruit pericarp. Innov. Food Sci. Emerg. Technol. 2009, 10, 413-419. [CrossRef]

12. Sudjaroen, Y.; Hull, W.E.; Erben, G.; Würtele, G.; Changbumrung, S.; Ulrich, C.M.; Owen, R.W. Isolation and characterization of ellagitannins as the major polyphenolic components of longan (Dimocarpus longan Lour.) seeds. Phytochemistry 2012, 77, 226-237. [CrossRef] [PubMed]

13. Yang, B.; Jiang, Y.; Shi, J.; Chen, F.; Ashraf, M. Extraction and pharmacological properties of bioactive compounds from longan (Dimocarpus longan Lour.) fruit-A review. Food Res. Int. 2011, 44, 1837-1842. [CrossRef]

14. Askjaer, P.; Galy, V.; Hannak, E.; Mattaj, I.W. Ran GTPase cycle and importins $\alpha$ and $\beta$ are essential for spindle formation and nuclear envelope assembly in living Caenorhabditis elegans embryos. Mol. Biol. Cell 2002, 13, 4355-4370. [CrossRef] [PubMed]

15. Bamba, C.; Bobinnec, Y.; Fukuda, M.; Nishida, E. The GTPase Ran regulates chromosome positioning and nuclear envelope assembly in vivo. Curr. Biol. 2002, 12, 503-507. [CrossRef]

16. Onuma, Y.; Nishihara, R.; Takahashi, S.; Tanegashima, K.; Fukui, A.; Asashima, M. Expression of the Xenopus GTP-binding protein gene Ran during embryogenesis. Dev. Genes Evol. 2000, 210, 325-327. [CrossRef] [PubMed]

17. Tian, Q.; Lin, Y.; Lai, Z.; Wang, T. Cloning and bioinformatic analysis of the promoters of DlRan3A and DlRan3B from embryogenic callus in Dimocarpus longan. Chin. J. Trop. Crops 2014, 35, 82-89.

18. Fang, Z.; Lai, C.; Zhang, Y.; Lai, Z. Molecular cloning, structural and expression profiling of DlRan genes during somatic embryogenesis in Dimocarpus longan Lour. SpringerPlus 2016, 5, 1-11. [CrossRef] [PubMed]

19. Ren, M.; Drivas, G.; D’Eustachio, P.; Rush, M.G. Ran/tc4: A small nuclear GTP-binding protein that regulates DNA synthesis. J. Cell Biol. 1993, 120, 313-323. [CrossRef] [PubMed]

20. Melchior, F.; Gerace, L. Two-way trafficking with Ran. Trends Cell Biol. 1998, 8, 175-179. [CrossRef]

21. Lai, Z.; Chen, C. Changes of endogenous phytohormones in the process of somatic embryogenesis in longan (Dimocarpus longan Lour.). Chin. J. Trop. Crops 2001, 23, 41-47.

22. Kim, S.-H.; Roux, S.J. An Arabidopsis Ran-binding protein, AtRanBP1c, is a co-activator of Ran GTPase-activating protein and requires the C-terminus for its cytoplasmic localization. Planta 2003, 216, 1047-1052. [PubMed]

23. Chen, M.; Du, X.; Zhu, Y.; Wang, Z.; Hua, S.; Li, Z.; Guo, W.; Zhang, G.; Peng, J.; Jiang, L. Seed fatty acid reducer acts downstream of gibberellin signalling pathway to lower seed fatty acid storage in Arabidopsis. Plant Cell Environ. 2012, 35, 2155-2169. [CrossRef] [PubMed]

24. Kępczyńska, E.; Zielińska, S. Regulation of Medicago sativa L. Somatic embryos regeneration by gibberellin $\mathrm{A}_{3}$ and abscisic acid in relation to starch content and $\alpha$-amylase activity. Plant Growth Regul. 2006, 49, $209-217$. [CrossRef] 
25. Kosová, K.; Vítámvás, P.; Prášil, I.T.; Renaut, J. Plant proteome changes under abiotic stress—Contribution of proteomics studies to understanding plant stress response. J. Proteom. 2011, 74, 1301-1322. [CrossRef] [PubMed]

26. Ralevski, A. The Interaction of Early Salt Stress-Induced 2 (ESI2) and the Ran G Protein in Arabidopsis; Concordia University: Irvine, CA, USA, 2013.

27. Xu, P.; Cai, W. RAN1 is involved in plant cold resistance and development in rice (Oryza sativa). J. Exp. Bot. 2014, 65, 3277-3287. [CrossRef] [PubMed]

28. Lai, Z.; Chen, C.; Zeng, L.; Chen, Z. Somatic embryogenesis in longan (Dimocarpus longan Lour.). In Somatic Embryogenesis in Woody Plants; Jain, S.M., Gupta, P., Newton, R., Eds.; Springer: Dordrecht, The Netherlands, 2000; Volume 67, pp. 415-431.

29. Lai, Z.; Chen, Z. Somatic embryogenesis of high frequency from longan embryogenic calli. J. Fujian Agric. Univ. 1997, 26, 271-276.

30. Lin, Y.-L.; Lai, Z.-X. Superoxide dismutase multigene family in longan somatic embryos: A comparison of $\mathrm{CuZn-SOD}, \mathrm{Fe}-\mathrm{SOD}$, and $\mathrm{Mn}$-SOD gene structure, splicing, phylogeny, and expression. Mol. Breed. 2013, 32, 595-615. [CrossRef]

31. Lescot, M.; Déhais, P.; Thijs, G.; Marchal, K.; Moreau, Y.; van de Peer, Y.; Rouzé, P.; Rombauts, S. Plantcare, a database of plant cis-acting regulatory elements and a portal to tools for in silico analysis of promoter sequences. Nucleic Acids Res. 2002, 30, 325-327. [CrossRef] [PubMed]

32. Sparkes, I.A.; Runions, J.; Kearns, A.; Hawes, C. Rapid, transient expression of fluorescent fusion proteins in tobacco plants and generation of stably transformed plants. Nat. Protoc. 2006, 1, 2019-2025. [CrossRef] [PubMed]

33. Chen, G.; Lu, L.; Lai, Z.; Pan, D. Extraction and purification of genomic DNA in Nai (Prunus salicina Lindl. var. cordata). Acta Agric. Univ. Jiangxiensis 2004, 26, 329-333.

34. Lin, Y.; Lai, Z. Reference gene selection for qPCR analysis during somatic embryogenesis in longan tree. Plant Sci. 2010, 178, 359-365. [CrossRef]

(C) 2016 by the authors; licensee MDPI, Basel, Switzerland. This article is an open access article distributed under the terms and conditions of the Creative Commons Attribution (CC-BY) license (http://creativecommons.org/licenses/by/4.0/). 